\title{
Impacto das mudanças climáticas na saúde pública: revisão integrativa
}

\author{
Impact of climate change on public health: an integrative review
}

Impacto del cambio climático en la salud pública: una revisión integradora

João Marcos Alves Pereira ${ }^{1 *}$, Luana Meireles Pecoraro ${ }^{1}$, Hélio Tavares de Oliveira Neto ${ }^{1}$, Fabíola Gabriellen de Barros Brito ${ }^{1}$, Francisco Edson Fagundes Neto ${ }^{1}$, Carliana Ingrid de Castro Silva ${ }^{1}$, Paulo Eduardo Soares Fonseca Filho ${ }^{1}$, Yahanna da Costa Anacleto Estrela ${ }^{1}$, Yoshyara da Costa Anacleto Estrela ${ }^{1}$, Milena Nunes Alves de Sousa ${ }^{1,2}$.

\section{RESUMO}

Objetivo: Evidenciar como as mudanças climáticas influenciam a saúde pública. Métodos: Foi adotada uma Revisão Integrativa de Literatura (RIL), em que foram selecionados os descritores: "climate change" e "public health". Os artigos selecionados estavam indexados nas seguintes bases de dados: Biblioteca Virtual em Saúde (BVS) e U.S National Library of Medicine and the National Institutes Health (PUBMED). Utilizou-se filtros de inclusão de acordo com a disponibilidade da base de dados. A partir disso, foram usados como critérios de exclusão os artigos que não condizem com a questão da pesquisa, publicados anteriores a 2015 e as repetições. Resultados: Constatou que as mudanças climáticas têm implicações sobre a saúde pública, pois diante dos assuntos enfatizados, as Doenças e a Criação de Políticas de Saúde Pública representaram $47,05 \%$ da amostra, cada, Entre os destaques de cada categoria, foram verificadas as Doenças Transmitidas por Vetores (17,65\%) e as Estratégias Governamentais e de Lideranças em saúde (41,18\%), respectivamente. Considerações finais: Em síntese, dentre as mudanças abordadas, temos como exemplo o aumento de temperatura, número crescente de vetores transmissores de doenças e poluição, que influenciam de forma direta na saúde da população, seja por meio de doenças ou pela diminuição da qualidade de vida dessas pessoas.

Palavras-chave: Vigilância em saúde pública, Alteração ambiental, Promoção da saúde.

\begin{abstract}
Objective: To highlight how climate change influences public health. Methods: An Integrative Literature Review (RIL) was adopted, in which descriptors were selected: "climate change" and "public health". The selected articles were indexed in the following databases: Virtual Health Library (VHL) and U.S National Library of Medicine and the National Institutes Health (PUBMED). Therefore, inclusion filters were used according to the availability of the database. From this, articles that do not match the research question, published prior to 2015 and repetitions were used as exclusion criteria. Results: He found that climate change has implications for public health, because in the light of the issues emphasized, Diseases and Public Health Policies represented $47.05 \%$ of the sample, each. Among the highlights of each category, Transmitted Diseases were verified by Vectors (17.65\%) and the Government and Leadership Strategies in health (41.18\%), respectively. Final considerations: In summary, among the changes addressed, we have as an example the increase in temperature, an increasing number of vectors that transmit diseases and pollution, which directly influence the health of the population, either through diseases or by decreasing quality of life. of these people.
\end{abstract}

Keywords: Public health surveillance, Environmental change, Health promotion.

${ }^{1}$ Centro Universitário de Patos (UNIFIP), Patos - PB. *E-mail: jm.al.ves@hotmail.com

${ }^{2}$ Faculdade São Francisco da Paraíba (FASP), Cajazeiras - PB.

SUBMETIDO EM: 7/2020

ACEITO EM: 8/2020

PUBLICADO EM: 11/2020

REAS/EJCH | Vol.12(11) | e4720 | DOI: https://doi.org/10.25248/reas.e4720.2020 


\section{RESUMEN}

Objetivo: Mostrar cómo el cambio climático influye en la salud pública. Métodos: Se adoptó una Revisión De la Literatura Integrativa (RIL), en la que se seleccionaron los descriptores: "cambio climático" y "salud pública". Los artículos seleccionados fueron indexados en las siguientes bases de datos: Biblioteca Virtual de Salud (VHL) y Biblioteca Nacional de Medicina de los Estados Unidos y los Institutos Nacionales de Salud (PUBMED). Los filtros de inclusión se utilizaron según la disponibilidad de la base de datos. A partir de esto, los criterios de exclusión fueron los artículos que no coinciden con la pregunta de investigación, publicados antes de 2015 y las repeticiones. Resultados: Se encontró que el cambio climático tiene implicaciones para la salud pública, ya que, habida cuenta de las cuestiones más destacadas, las enfermedades y la creación de políticas de salud pública representaron el $47,05 \%$ de la muestra, cada una de las que destaca cada categoría, se verificaron las enfermedades transmitidas por vectores $(17,65 \%)$ y estrategias gubernamentales y de liderazgo $(41,18 \%)$, respectivamente. Consideraciones finales: En resumen, entre los cambios abordados, tenemos como ejemplo el aumento de la temperatura, el aumento del número de vectores que transmiten enfermedades y contaminación, que influyen directamente en la salud de la población, ya sea a través de enfermedades o reduciendo la calidad de vida de estas personas.

Palabras clave: Vigilancia de la salud pública, Cambio ambiental, Promoción de la salud.

\section{INTRODUÇÃO}

As mudanças climáticas globais são consideradas uma grande ameaça aos sistemas humanos e naturais e, nos últimos anos, a atenção dada ao assunto por parte da mídia e de agendas políticas e científicas internacionais tem aumentado (WEBER EU e STERN PC, 2011; CLAYTON S, et al., 2015).

Nesse cenário, as evidências científicas sobre os impactos provocados pelo homem ao meio ambiente são cada vez mais conhecidas e consensuais (GIFFORD R, 2008; WEBER EU e STERN PC, 2011; CLAYTON $S$, et al., 2015). Com isso, a crescente preocupação com o meio ambiente, que ganhou destaque na década de 1970, trouxe inúmeras contribuições no que diz respeito a sua proteção e preservação (CLAYTON S, et al., 2015; BARCELLOS C, et al., 2016).

Os impactos ambientais e sua influência sobre a saúde não é uma questão nova. Em 1990, a Organização Mundial de Saúde (OMS) publicou o primeiro informe científico relacionado as mudanças climáticas e saúde (WEBER EU e STERN PC, 2011). Em 1995, foi realizada uma conferência em Washington, em que a Organização Panamericana de Saúde (OPAS) apresentou esse tema para a Academia Nacional de Ciências dos Estados Unidos. Desde então diversos estudos e evidências comprovam essa relação e alertam sobre os efeitos sobre a saúde, apontando para uma maior consciência e preparação no enfrentamento às ameaças e aos efeitos que surgirem (WEBER EU e STERN PC, 2011; CLAYTON S, et al., 2015).

É notório, também, que a urbanização tem sido um fator determinante para a mudança do perfil epidemiológico e da situação de saúde, especialmente nas grandes cidades, em que as condições de vida vêm se deteriorando, seja pelo resultado direto da poluição e/ou ocupação pela industrialização, ou pela pressão demográfica sobre o meio ambiente, ou ainda pelas grandes desigualdades sociais, ampliadas em meio aos dois processos citados (SOUZA CL e ANDRADE CS, 2014; BARCELLOS C, et al., 2016).

Os impactos causados ao meio ambiente não se confinam apenas aos limites de determinados países ou regiões, atualmente, já ultrapassam fronteiras e, rotineiramente, atingem todas as regiões do mundo (WEBER EU e STERN PC, 2011; CLAYTON S, et al., 2015).

Corrobora com a assertiva Barcellos C, et al. (2016), ao afirmar que essas transformações no ecossistema podem promover uma variedade de impactos em diversas áreas, sobretudo na saúde humana e bem-estar da população. Cenários de variações extremas, desde as ondas de calor às de frio ou furacões, tempestades, inundações. No entanto, seu impacto principal é o indireto, mediado por processos ambientais, como alterações nos ecossistemas e biodiversidade (WEBER EU e STERN PC, 2011). 
Todavia, os fatores sociais podem reduzir ou ampliar os impactos na saúde populacional de acordo com a apropriação diferencial dos recursos disponibilizados. Então é nesse sentido que pesquisas precisam evidenciar o problema para uma tentativa de capacitar os indivíduos, grupos e comunidade a responderem potenciais perigos desencadeados por eventos relacionados às mudanças ambientais e climáticas (ALPINO TA, et al., 2016).

No Brasil, apesar de ainda ser detentor de grande biodiversidade e disponibilidade de recursos naturais, vem apresentando altos índices de devastação e esgotamento desses recursos, o que tem contribuído para grandes mudanças climáticas (PACHAURI RK e MAYER L, 2014; ALPINO TA, SENA ARM e FREITAS CM, et al., 2016).

Ao observar o Quinto Relatório do Painel Intergovernamental das Mudanças Climáticas (IPCC), espera-se uma elevação na tempera da superfície terrestre entre os anos entre os anos de 2016 a 2035 entre 0,3 a $0,7^{\circ} \mathrm{C}$. Ademais, foi possível concluir, a partir do citado relatório, que as intercorrências sobre a saúde humana não e sua intercessão com as alterações climáticas não foram adequadamente explicitados e nem quantificados (PACHAURI RK e MAYER L, 2014).

Além das mudanças climáticas outros fatores são relacionados a maior dimensão de impactos ambientes, dentre eles, crescimento populacional, baixas condições econômicas e sanitárias da população, falta de infraestrutura familiar que contribui para a desorganização ambiental, geração de efluentes e descarte inadequado do lixo, bem como a falta de fiscalização e de ações públicas por parte das entidades governamentais competentes (LADIN R e GIATTI LL, 2014; ALPINO TA, SENA ARM e FREITAS CM, et al., 2016).

A partir do quadro elucidado, verifica-se a atualidade do objeto de estudo, o que indica o seu crescente interesse entre os muitos pesquisadores, uma vez que é possível indicar estratégias para resolução do problema, tais como a efetivação de ações prioritárias de vigilância em saúde e em políticas públicas para o monitoramento e controle dos impactos (PACHAURI RK e MAYER L, 2014). Por isso, há uma necessidade de evidenciar a emergência de romper uma espécie de fronteira entre o presente e o futuro, uma vez a existência da imprevisibilidade e da incerteza relacionadas às questões ambientais, especialmente as mudanças climáticas e à vulnerabilidade socioambiental (LADIN R e GIATTI LL, 2014; PACHAURI RK e MAYER L, 2014).

Ademais, o presente trabalho tem como objetivo demonstrar a relação atual entre saúde pública e as mudanças climáticas, de forma a esclarecer ou evidenciar de que forma as políticas de saúde pública podem contribuir positivamente no controle e monitoramento desses impactos ambientais.

\section{MÉTODOS}

Esse artigo é uma Revisão Integrativa de Literatura (RIL) com o tema "mudanças climáticas e saúde pública". A RIL é um método de pesquisa que fornece informações amplas sobre uma determinada questão, e para isso, utiliza como ferramenta uma questão central como embasamento para pesquisas e posterior condensação dos resultados (RIBEIRO OMP, MARTINS MMF e TRONCHIN DMR, 2016). Consiste em um estudo qualitativo em que são adotados critérios bem estabelecidos para uma pesquisa nas bases de dados e, posteriormente, apresentação dos resultados (RIBEIRO OMP, et al., 2016; SOUZA AM e PEGORARO RF, 2017).

Após a escolha do tema, é essencial definir uma questão de pesquisa, pois é ela quem vai orientar a predileção dos artigos. Diante disso, foi definida como pergunta da pesquisa: quais os impactos das mudanças climáticas na saúde pública?

Diante disso, para fazer uma pesquisa dentro dos padrões, é necessário identificar os descritores que irão ser usados nas bases de dados para que os artigos corretos estejam disponíveis. Para isso, foram selecionados dois Descritores Controlados em Ciências da Saúde (DeCS) na língua inglesa: "climate change" e "public health". O operador booleano utilizado foi and com objetivo de relacionar os termos. Na busca dos artigos selecionaram-se os que estavam indexados nas seguintes bases de dados: Biblioteca Virtual em Saúde (BVS) e U.S National Library of Medicine and the National Institutes Health (PUBMED). 
Para a predileção e filtragem dos artigos, foram utilizados os seguintes filtros de inclusão de acordo com a disponibilidade da base de dados: estudos realizados com humanos, publicados nos últimos 5 anos, texto completo e presença dos descritores no título. A partir disso, foram usados como critérios de exclusão os artigos que não condiziam com a questão norteadora da pesquisa, publicados anteriores ao ano de $2015 \mathrm{e}$ os estudos repetidos (Figura 1).

Figura 1 - Processo de seleção dos artigos sobre o objeto de estudo.

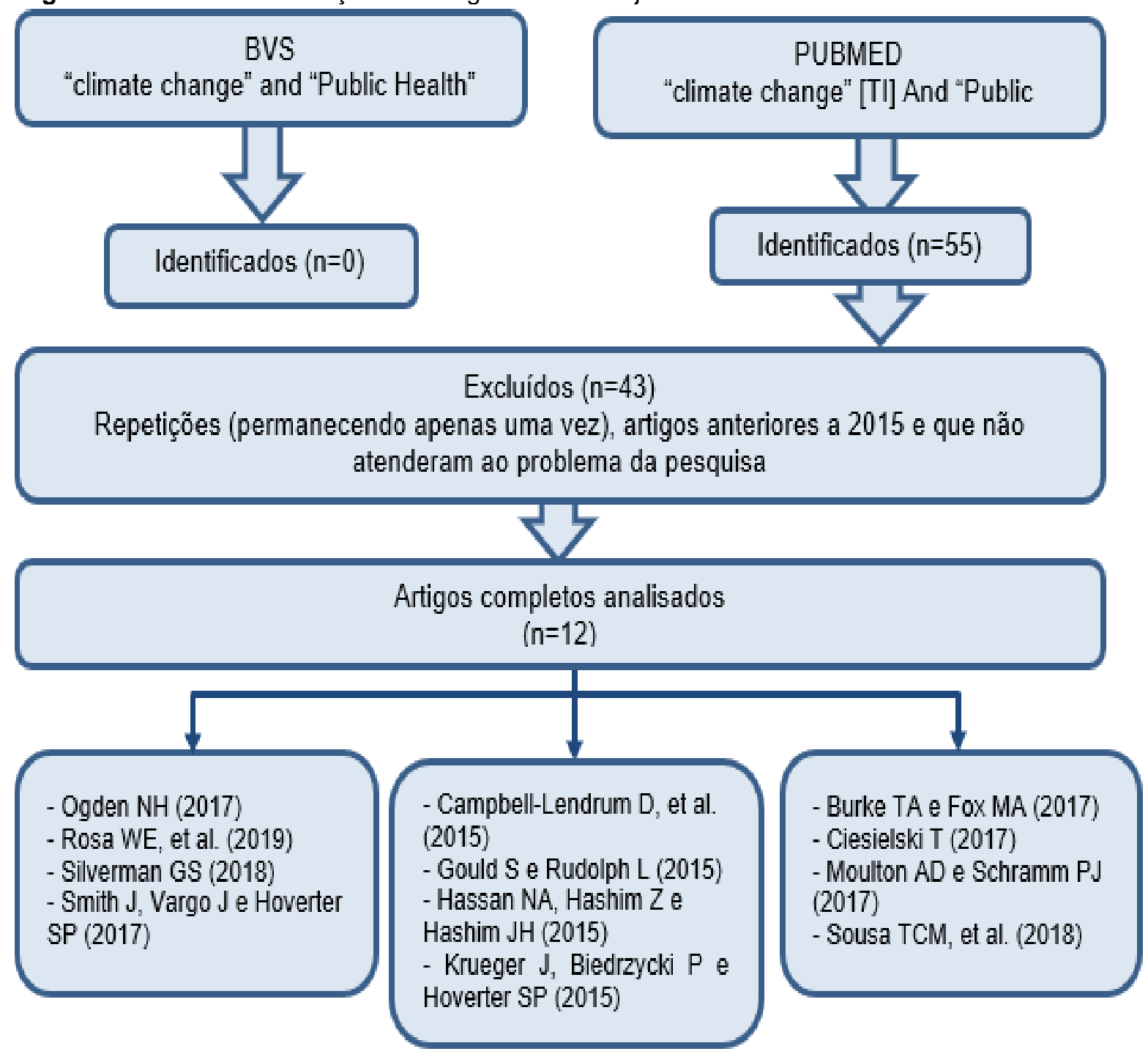

Fonte: Pereira JMA, et al., 2020.

Sequencialmente, com a obtenção de 55 artigos, foi feita uma leitura de todos os artigos escolhidos e, posteriormente, foram selecionados de acordo com os seguintes critérios: autores, título do artigo, base de dados, revista, idioma, país, método, população de estudo e os principais achados. A amostra foi composta por 12 artigos. Ainda, realizou-se uma categorização desses artigos diante de 3 categorias para melhor elaboração dos resultados e discussão.

\section{RESULTADOS E DISCUSSÃO}

Dos 12 artigos selecionados nesta revisão, 100\% ( $n=12)$ estavam disponíveis da base de dados do PUBMED, $41,67 \%(n=5)$ são do ano de 2017, 91,67\% ( $n=11)$ foram publicados em inglês, $58,33 \%(n=7)$ publicados nos Estados Unidos e, por fim, $16,67 \%(n=2)$ foram encontrados na revista APJH Climate Change e $83,4 \%(n=10)$ foram feitos seguindo a metodologia de revisão narrativa (Quadro 1). 


\section{Revista Eletrônica Acervo Saúde / Electronic Journal Collection Health | ISSN 2178-2091}

Quadro 1 - Caracterização dos artigos selecionados quanto aos autores, ano, título, revista, idioma, país e método.

\begin{tabular}{|c|c|c|c|c|c|}
\hline Autores/Ano & Título do Artigo & Revista & Idioma & País & Método \\
\hline $\begin{array}{l}\text { Burke TA e Fox MA } \\
(2017)\end{array}$ & $\begin{array}{l}\text { Global to Local: Public Health on the Front Lines } \\
\text { of Climate Change }\end{array}$ & AJPH climate change & Inglês & Estados Unidos & Revisão Narrativa \\
\hline $\begin{array}{l}\text { Campbell-Lendrum D, et } \\
\text { al. (2015) }\end{array}$ & $\begin{array}{l}\text { Climate change and vector-borne diseases: what } \\
\text { are the implications for public health research } \\
\text { and policy? }\end{array}$ & The Royal Society Publishing & Inglês & Suíça & Revisão narrativa \\
\hline Ciesielski T (2017) & $\begin{array}{c}\text { Climate Change and Public Health: A Small } \\
\text { Frame Obscures the Picture }\end{array}$ & $\begin{array}{c}\text { New Solutions: a Journal of } \\
\text { Inveronmetal and Occupational } \\
\text { Health Policy }\end{array}$ & Inglês & Estados Unidos & Revisão narrativa \\
\hline $\begin{array}{l}\text { Gould S e Rudolph L } \\
\qquad(2015)\end{array}$ & $\begin{array}{l}\text { Challenges and Opportunities for Advancing } \\
\text { Work on Climate Change and Public Health }\end{array}$ & $\begin{array}{l}\text { International journal of } \\
\text { environmental research and } \\
\text { public health }\end{array}$ & Inglês & Califórnia & $\begin{array}{l}\text { Qualitativa (entrevista } \\
\text { semi-estruturadas em } \\
\text { profundidade) }\end{array}$ \\
\hline Hassan NA, et al. (2015) & $\begin{array}{l}\text { Impact of Climate Change on Air Quality and } \\
\text { Public Health in Urban Areas }\end{array}$ & $\begin{array}{l}\text { Asia-Pacific Journal of Public } \\
\text { Health }\end{array}$ & Inglês & Malásia & Revisão narrativa \\
\hline Krueger J, et al. (2015) & $\begin{array}{c}\text { Human Health Impacts of Climate Change: } \\
\text { Implications for the Practice and Law of Public } \\
\text { Health }\end{array}$ & Journal of Law, Medicine \& Ethics & Inglês & Estados Unidos & Revisão narrativa \\
\hline $\begin{array}{l}\text { Moulton AD e Schramm } \\
\text { PJ (2017) }\end{array}$ & $\begin{array}{c}\text { Climate Change and Public Health Surveillance: } \\
\text { Toward a Comprehensive Strategy }\end{array}$ & $\begin{array}{l}\text { Journal of public health } \\
\text { management and practice: } \\
\text { JPHMP }\end{array}$ & Inglês & Estados Unidos & Revisão narrativa \\
\hline Ogden NH (2017) & $\begin{array}{l}\text { Climate change and vector-borne diseases of } \\
\text { public health significance }\end{array}$ & FEMS microbiology letters & Inglês & Canadá & Revisão narrativa \\
\hline Rosa WE, et al. (2019) & $\begin{array}{c}\text { Climate change and health consequences: } \\
\text { Engaging public health nursing within the } \\
\text { framework of the United Nations Sustainable } \\
\text { Development Goals }\end{array}$ & Public Health Nurs & Inglês & Estados Unidos & Revisão narrativa \\
\hline Silverman GS (2018) & $\begin{array}{c}\text { Systematic Lack of Educational Preparation in } \\
\text { Addressing Climate Change as a Major Public } \\
\text { Health Challenge }\end{array}$ & AJPH climate change & Inglês & Estados Unidos & Revisão Narrativa \\
\hline Smith J, et al. (2017) & Climate Change and Public Health Policy & $\begin{array}{l}\text { The Journal of Law, Medicine \& } \\
\text { Ethics }\end{array}$ & Inglês & Estados Unidos & Revisão narrativa \\
\hline Sousa TCM, et al. (2018) & $\begin{array}{l}\text { Doenças sensíveis ao clima no Brasil e no } \\
\text { mundo: revisão sistemática }\end{array}$ & Rev Panam Salud Publica & Português & Brasil & $\begin{array}{l}\text { Revisão sistemática } \\
\text { de metodologia prisma }\end{array}$ \\
\hline
\end{tabular}

Fonte: Pereira JMA, et al., 2020.

$\overline{\text { REAS/EJCH | Vol.12(11) | e4720 | DOI: https://doi.org/10.25248/reas.e4720.2020 Página } \mathbf{5} \text { de } \mathbf{9}}$ 
Diante dos assuntos enfatizados, as Doenças representaram $47,05 \%$ ( $n=8)$ da amostra, com destaque para as Doenças Transmitidas por Vetores (17,65\%; $n=3)$. Além disso, Criação de Políticas de Saúde Pública também foram citadas em (47,05\%), sendo Estratégias Governamentais e de Lideranças em saúde (41,18\%; $\mathrm{n}=8$ ) dos artigos (Quadro 2).

Embora tenha havido equivalência de artigos encontrados para as duas categorias, a subcategoria "estratégias governamentais e de lideranças em saúde" tem uma maior representatividade dentre as publicações selecionadas.

Quadro 2 - Caracterização dos artigos selecionados quanto as categorias temáticas e subcategorias.

\begin{tabular}{|c|c|c|c|c|}
\hline Categorias & Subcategorias & Autores/anos & $\mathbf{N}$ & $\%$ \\
\hline \multirow{5}{*}{ Doenças } & $\begin{array}{l}\text { Doenças transmitidas } \\
\text { por vetores }\end{array}$ & $\begin{array}{l}\text { Krueger J, et al. (2015) } \\
\text { Ogden NH (2017) } \\
\text { Sousa TCM, et al. (2018) }\end{array}$ & 3 & 17,65 \\
\hline & $\begin{array}{c}\text { Doenças sensíveis ao } \\
\text { clima }\end{array}$ & Campbell-Lendrum D, et al. (2015) & 1 & 5,88 \\
\hline & Doenças metabólicas & Rosa WE, et al. (2019) & 1 & 5,88 \\
\hline & $\begin{array}{c}\text { Doenças } \\
\text { cardiovasculares }\end{array}$ & $\begin{array}{c}\text { Ciesielski T (2017) } \\
\text { Sousa TCM, et al. (2018) }\end{array}$ & 2 & 11,76 \\
\hline & Doenças respiratórias & Ciesielski T (2017) & 1 & 5,88 \\
\hline \multirow[t]{2}{*}{$\begin{array}{c}\text { Criação de } \\
\text { políticas } \\
\text { de saúde } \\
\text { pública }\end{array}$} & $\begin{array}{l}\text { Estratégias } \\
\text { governamentais e de } \\
\text { lideranças em saúde }\end{array}$ & $\begin{array}{c}\text { Burke TA e Fox MA (2017) } \\
\text { Hassan NA, et al. (2015) } \\
\text { Campbell-Lendrum D, et al. (2015) } \\
\text { Moulton AD e Schramm PJ (2017) } \\
\text { Rosa WE, et al. (2019) } \\
\text { Silverman GS (2018) } \\
\text { Smith J, et al. (2017) }\end{array}$ & 7 & 41,18 \\
\hline & Barreiras institucionais & Gould S e Rudolph L (2015) & 1 & 5,88 \\
\hline $\begin{array}{l}\text { Poluição } \\
\text { das águas }\end{array}$ & $\begin{array}{c}\text { Contaminação dos } \\
\text { peixes }\end{array}$ & Ciesielski T (2017) & 1 & 5,88 \\
\hline \multicolumn{3}{|c|}{ Total } & 17 & 100 \\
\hline
\end{tabular}

Fonte: Pereira JMA, et al., 2020.

As alterações climáticas podem causar impactos sobre a saúde humana de forma direta, como no caso de eventos extremos como secas e estiagens, enchentes, incêndios, deslizamento de terra, furacão, tsunamis e fortes ondas de calor, que podem causar mortes; e de forma indireta sendo provocado por alterações de ecossistemas e de ciclos de biológicos, que podem contribuir para o aumento da incidência de doenças infecciosas, causadas por vetores e também doenças não-transmissíveis com desnutrição (PACHAURI RK e MAYER L, 2014; OGDEN NH, 2017). Ademais, alterações de temperatura e umidade e a exposição a poluentes atmosféricos podem provocar piora das doenças respiratórias (BUCHANAN S, et al., 2014; CAMPBELL-LENDRUM D, 2015).

As doenças transmitidas por vetores, segundo a Organização Mundial da Saúde (OMS, 2020), como a dengue, febre amarela, malária, esquistossomose, leishmaniose, doença de Chagas e peste representam mais de $17 \%$ das doenças infecciosas no mundo, causando mais de 700 mil mortes por ano. Sabendo disso, deve-se destacar que os riscos dessas doenças são intrinsecamente sensíveis às mudanças climáticas, como é relatado por Ogden NH (2017), ou seja, o clima tem impactos indiretos na ocorrência do vetor, determinando as qualidades do seu hábitat e a sua sobrevivência, sendo assim fatores importantes para determinar a propagação das doenças transmitidas por insetos.

No Brasil, apesar do aumento da cobertura dos serviços de abastecimento de água, que atinge $91,3 \%$ da população da zona urbana, há um alto índice de doenças de veiculação hídrica, sendo destacadas como as mais incidentes, esquistossomose, hepatite A, leptospirose e gastroenterites (OGDEN NH, 2017). Isso pode ser explicado pelos impactos provocados pelas mudanças climáticas, agravando as enchentes ou secas e comprometendo a qualidade e o acesso à água (PACHAURI RK e MAYER L, 2014). 
Em função dos impactos causados ao meio ambiente, muitas doenças sofrem influências negativas, de modo que as contínuas taxas de aumento da temperatura e a variação climática ameaçam minar o recente progresso global contra essas patologias (BUCHANAN S, et al., 2014). De acordo com Campbell-Lendrum D (2015), as mudanças climáticas, promovidas por fatores antropogênicos, de longo prazo, interagem com a variabilidade natural e influenciam na disseminação de doenças transmitidas por vetores.

Dentre esses fatores, segundo os autores, tem-se o aumento de temperatura, mudanças nos padrões geográficos de precipitação, aumento da variabilidade climática e aumento da frequência e severidade de eventos climáticos extremos, todas elas alterando o equilíbrio da relação homem e natureza, contribuindo para os atuais problemas de saúde pública (CAMPBELL-LENDRUM D, 2015).

Dentre os impactos provocados a saúde destaca-se também as possíveis alterações causadas na produção agrícola que prejudicam o abastecimento e segurança dos alimentos, reduzindo sua disponibilidade e que, consequentemente, pode causar desnutrição e influencia negativamente no crescimento e desenvolvimento das crianças (PACHAURI RK e MAYER L, 2014). Além de intoxicações por agrotóxicos que provocam alterações na quantidade e qualidade dos alimentos e da água potencializando a incidência de patologias diarreicas e outras doenças de veiculação hídrica citadas anteriormente (PACHAURI RK e MAYER L, 2014; CAMPBELL-LENDRUM D, 2015; OGDEN NH, 2017).

É de conhecimento que as mudanças climáticas, como a queima de combustíveis fósseis, muito utilizados para geração de energia elétrica e combustível para automotores, afetam a saúde humana de forma direta, sendo relatada por Ciesielski T (2017) como um fator preponderante para intensificar fatores respiratórios, como asma e alergias, assim como doenças cardiovasculares. Buchanan S, et al. (2014) mostraram que a queima de carvão e de materiais poluentes, liberam partículas como dióxido de carbono e enxofre, que levam a inflamação, citotoxicidade e morte celular, contribuindo para gerar as comorbidades ou agravá-las e, se expostos em longo prazo, levar a óbito.

A OMS (2006) estima que em todo o mundo, $5 \%$ das mortes cardiopulmonares são desencadeadas pela aspiração de micropartículas de poluentes disseminadas pelo ar. Ademais, Gan WQ, et al. (2011) também relatam que a exposição prolongada a esses fatores aumenta os riscos de doenças coronarianas, de hospitalização e de morbimortalidade.

Sabendo-se que a queima de poluentes também afeta a temperatura ambiental, Sousa TCM, et al. (2018) analisou a mudança climática, mais especificamente o aumento da temperatura que tem influência sobre os ventos, chuvas e demais eventos climáticos, como o agravamento tanto de patologias cardiovasculares e pulmonares, como também de doenças metabólicas devido à pressão atmosférica, temperatura e umidade da região.

Além disso, Rosa WE, et al. (2019) mostraram que essas alterações climáticas, as quais afetam a saúde humana, advêm justamente da crescente população e da destruição progressiva do meio ambiente, expondo a necessidade de estimular os esforços de da população e das entidades governamentais para promoção de saúde pública, com ações voltados para o meio ambiente e sua preservação, para minimizar as mudanças climáticas e os malefícios que causam a saúde da população (SANTOS LTS e JESUS TB, 2014; SOUZA AKR, et al., 2018).

No entanto, constata-se que a degradação do meio ambiente também tem implicações negativas sobre o ecossistema e outras espécies, além do homem. Os efeitos combinados do desmatamento e aquecimento global são os principais problemas que ameaçam, por exemplo, a sobrevivência e a conectividade dos animais aquáticos, como os peixes, e causam prejuízos a saúde dos indivíduos que se alimentam dessas espécies (HURD LE, et al., 2016). De acordo com Ciesielski T (2017), algumas dessas questões já são muito bem descritas e vêm prejudicando nossa saúde há anos. Por exemplo, a queima de carvão tem acumulado um tipo de mercúrio a níveis tóxicos como o metilmercúrio, que contamina os rios e os peixes e, tem gerado problemas de saúde pela ingestão desses animais contaminados.

Segundo a OMS (2008), episódios de intoxicação com características epidêmicas foram associados principalmente aos compostos orgânicos de mercúrio, alguns deles decorrentes da ingestão de peixes contaminados por metilmercúrio. O problema da contaminação do ecossistema por metais como o mercúrio 
atinge grandes extensões territoriais e afeta sistemas fluviais, estuarinos e marítimos, sendo observado em diferentes partes do mundo como consequência da expansão industrial (CIESIELSKI T, 2017; SOUZA AKR, MORASSUTI CY e DEUS WB, 2018). Nesse contexto, mais uma vez destaca-se a importância de medidas para minimizar os impactos que o homem causa ao meio ambiente (SANTOS LTS e JESUS TB, 2014; LIMA DP, et al., 2015; SOUZA AKR, et al., 2018).

Sabe-se que as políticas públicas são de fundamental importância quando se trata das mudanças climáticas e seus impactos na saúde da população. Smith J, et al. (2017) apontam estudos de divulgação científica em relação às mudanças climáticas, como o Painel Intergovernamental de Mudanças Climáticas (IPCC) e o Relatório de Comissão da Lancet "Health and Climate Change", que servem de base para a formulação de políticas de saúde pública objetivando orientar a população para tais mudanças. Para a criação de estratégias de qualidade, Moulton AD e Schramm PJ (2017) afirmam que são necessários planos abrangentes de forma a criar uma vigilância em saúde de forma multissetorial que tenha capacidade de atender a todos os níveis de assistência e à população.

O setor saúde necessita tomar medidas e intervenções que objetivem a redução dos impactos provocados ao meio ambiente, através de discussões Inter setoriais visando à proteção da saúde e de investimentos em projetos voltados para as populações que estão localizadas em regiões mais vulneráveis (MOULTON AD e SCHRAMM PJ, 2017), visto que as mudanças climáticas parecem estar relacionadas às questões de vulnerabilidade individual e coletiva, devendo sempre considerar o perfil socioeconômico de cada população (BURKE TA e FOX MA, 2017; ROSA WE, et al., 2019).

Além disso, Burke TA e Fox MA (2017) também demonstram a necessidade de uma mitigação de esforços globais e dos setores sociais para que haja um estímulo para pesquisas e projetos que auxiliam no combate ao surgimento de doenças e quaisquer outros problemas de saúde que venham a surgir com as mudanças climáticas. Nesse contexto, os profissionais da saúde são essenciais para o processo de justiça ambiental, como forma de elaboração de planos de ação para ajudar na redução dos impactos ambientais e favorecer a saúde pública (ROSA WE, et al., 2019). No entanto, segundo Silverman GS (2018) ainda existe falta de preparação desses profissionais para a realização dessas estratégias.

Por mais que se saiba dos impactos das mudanças climáticas na saúde pública, existem ainda barreiras que impedem a efetivação dos profissionais da área no combate (SILVERMAN GS, 2018). É visto que há ainda uma falta de investimentos, lideranças e de capacidade de trabalho para essa efetivação, seja pela falta de um treinamento adequado ou pela falta desses profissionais não terem a ação de tomar lideranças e exporem opiniões públicas sobre o assunto (GOULD S e RUDOLPH L, 2015; SILVERMAN GS, 2018).

Em concordância, Hassan NA, et al. (2015) afirma que se torna um desafio para a formação de políticas que minimizem os impactos das mudanças climáticas é a falta de um conhecimento sobre a vulnerabilidade em relação a tais alterações do clima e também a falta de integração entre a sociedade em geral e a parcela da população interessada no problema.

As limitações desta revisão implicam no fato de que há poucos artigos originais que destacam a avaliação do impacto do homem no meio ambiente. A maior parte dos artigos científicos pesquisados está publicada em inglês e discute cenários dos Estados Unidos e países europeus. Além disso, nenhum dado primário foi incluído neste estudo devido ao tempo e restrições financeiras.

\section{CONSIDERAÇÕES FINAIS}

Em síntese, os achados demonstram a inter-relação entre as mudanças climáticas e suas implicações sobre a saúde pública. Dentre as transformações contempladas tem-se, como exemplos, o aumento de temperatura, o número crescente de vetores transmissores de doenças e a poluição, os quais influenciam de forma direta a saúde humana, seja por meio de doenças ou pela diminuição da qualidade de vida dessas pessoas. Portanto, as alterações climáticas afetam de forma negativa a saúde e são necessárias políticas públicas que procurem o engajamento de líderes políticos e de Organizações Não governamentais, bem como de profissionais da saúde à formulação de estratégias de prevenção e de preparação da população para essas mudanças, havendo necessidade de "apropriação", para reduzir ao máximo os impactos via ambiente e saúde pública que, de outra maneira, serão inevitáveis. 


\section{REFERÊNCIAS}

1. ALPINO TA, et al. Desastres relacionados à seca e saúde coletiva - uma revisão da literatura científica. Ciência \& Saúde Coletiva. 2016;21(1):809-820.

2. BARCELLOS $\mathrm{C}$, et al. An observatory to gather and disseminate information on the health-related effects of environmental and climate change. Revista Panamericana de Salud Pública. 2016;40(1):167-173.

3. BELL ML, et al. The avoidable health effects of air pollution in three Latin American cities: Santiago, Sao Paulo, and Mexico City. Environmental research. 2006;100(3):431-440.

4. BUCHANAN S, et al. Beyond black lung: scientific evidence of health effects from coal use in electricity generation. Journal Of Public Health Policy. 2014;35(3):266-277.

5. BURKE TA, FOX MA. Global to local: public health on the front lines of climate change. Ajph Editorials. 2018; 108(1):7475.

6. CAMPBELL-LENDRUM D, et al. Climate change and vector-borne diseases: what are the implications for public health research and policy? Philosophical Transactions of the Royal Society B: Biological Sciences. 2015; 370(552): $345-349$.

7. CIESIELSKI T. Climate change and public health: a small frame obscures the picture. New Solutions: a Journal of Inveronmetal and Occupational Health Policy. 2017;27(1):8-11.

8. CLAYTON S, et al. Psychological research and global climate change. Nature Climate Change. 2015;5(1):640-646.

9. GAN WQ, et al. Long-Term Exposure to Traffic-Related Air Pollution and the Risk of Coronary Heart Disease Hospitalization and Mortality. Environmental Health Perspectives. 2011;119(4):501-507.

10. GIFFORD R. Psychology's essential role in alleviating the impacts of climate change. Canadian Psychology. 2008;49(4):273-280.

11. GOULD S, RUDOLPH L. Challenges and opportunities for advancing work on climate change and public health. International journal of environmental research and public health. 2015;12(12):15649-15672.

12. HASSAN NA, et al. Impact of climate change on air quality and public health in urban areas. Asia Pacific Journal of Public Health. 2016;28(2):38-48.

13. HURD LE, et al. Amazon floodplain fish communities: habitat connectivity and conservation in a rapidly deteriorating environment. Biological Conservation. 2016;195(1):118-127.

14. KRUEGER J, et al. Human health impacts of climate change: implications for the practice and law of public health. The Journal of Law, Medicine \& Ethics. 2015;43(1):79-82.

15. LANDIN R, GIATTI LL. Política de mudança do clima no município de São Paulo, Brasil: reflexividade e permeabilidade do Setor Saúde. Ciência \& Saúde Coletiva. 2014;19(1):4149-4156.

16. LIMA DP, et al. Contaminação por metais pesados em peixes e água da bacia do rio Cassiporé, Estado do Amapá, Brasil. Acta Amazonica. 2015;45(4):405-414.

17. MOULTON AD, SCHRAMM PJ. Climate change and public health surveillance: toward a comprehensive strategy. Journal of public health management and practice: JPHMP. 2017; 23(6): 618-626.

18. OGDEN NH. Climate change and vector-borne diseases of public health significance. FEMS microbiology letters. 2017;364(19):1-8.

19. PACHAURI RK, MAYER L. Intergovernmental Panel on Climate Change. Climate change 2014: synthesis report. Genebra: Intergovernmental Panel on Climate Change; 2015.

20. REZENDE JM. A viagem científica de Neiva e Penna: roteiro para os estudos das doenças do sertão. História, Ciências, Saúde-Manguinhos. 2009;16(1): 265-288.

21. RIBEIRO OMP, et al. Modelos de prática profissional de enfermagem: revisão integrativa da literatura. Rev. Enf. Ref. 2016;4(10):125-133.

22. ROSA WE, et al. Climate change and health consequences: Engaging public health nursing within the framework of the United Nations Sustainable Development Goals. Public Health Nursing. 2019;36(2):107-108.

23. SANTOS LTS, JESUS TB. Caracterização de metais pesados das águas superficiais da bacia do Rio Subaé (Bahia). Geochimica Brasiliensis. 2014;28(2):137-148.

24. SILVERMAN GS. Systematic Lack of Educational Preparation in Addressing Climate Change as a Major Public Health Challenge. American journal of public health. 2019;109(2):242-243.

25. SMITH J, et al. Climate change and public health policy. The Journal of Law, Medicine \& Ethics. 2017;45(1): 82-85.

26. SOUSA TCM, et al. Doenças sensíveis ao clima no Brasil e no mundo: revisão sistemática. Revista Panamericana de Salud Pública. 2018;42(1):1-10.

27. SOUZA AKR, et al. Poluição do ambiente por metais pesados e utilização de vegetais como bioindicadores. Acta Biomédica Brasiliensia. 2018;9(3): 95-106.

28. SOUZA CL, ANDRADE CSa. Saúde, meio ambiente e território: uma discussão necessária na formação em saúde: uma discussão necessária na formação em saúde. Ciência \& Saúde Coletiva. 2014; 19(10):4113-4122.

29. WEBER EU, STERN PC. Public understanding of climate change in the United States. American Psychologist. 2011;66(4):315-328.

30. WORLD HEALTH ORGANIZATION. Guidance for identifying populations at risk from mercury exposure. Geneva: WHO; 2008.

31. WORLD HEALTH ORGANIZATION. Air Quality Guidelines: Global Update for 2005. Copenhagen: WHO; 2006.

32. SOUZA AM, PEGORARO RF. O psicólogo na UTI neonatal: revisão integrativa de literatura. Sau. \& Transf. Soc. 2017;8(1):117-128. 